\title{
LETTER \\ Apps at Hand: Personalized Live Homescreen Based on Mobile App Usage Prediction*
}

\author{
Xiao XIA ${ }^{\dagger, \dagger \dagger a)}$, Xinye LIN ${ }^{\dagger \dagger}$, Nonmembers, Xiaodong WANG ${ }^{\dagger}$, Member, Xingming ZHOU ${ }^{\dagger}$, \\ and Deke GUO ${ }^{\dagger}$, Nonmembers
}

\begin{abstract}
SUMMARY To facilitate the discovery of mobile apps in personal devices, we present the personalized live homescreen system. The system mines the usage patterns of mobile apps, generates personalized predictions, and then makes apps available at users' hands whenever they want them. Evaluations have verified the promising effectiveness of our system. key words: mobile app, personalized pattern, usage prediction, mobile app discovery, personalized live homescreen
\end{abstract}

\section{Introduction}

With the huge expansion of mobile devices in recent years, the development of mobile apps has undergone a tremendous growth. Such a tendency results in the continuous installations and frequent updates of apps on personal mobile devices. This eventually gives rise to the essential app discovery problem for mobile users. While the screen spaces of a device are rapidly consumed with so many apps installed, it becomes much more difficult for users to find the app they want among screens of apps. Although popular mobile operating systems provide multiple homescreens for users to customize shortcuts of apps for quick accessing, there are still lots of apps lying quietly in the background. Users often have to scroll through screens of apps just for discovering and accessing one. Such a problem significantly hurts the experiences of users, but it has attracted little attention from the research community.

To address such a challenge, we present the Personalized Live Homescreen (PLH) system that implements a novel "live homescreen" in a "personalized" way. The main efforts and contributions of this paper are as follows:

1. We originally propose the concept of live homescreen to tackle the app discovery problem of mobile devices. The live homescreen differs from the traditional homescreen by automatically predicting and displaying the apps that are of interest to a user, instead of always showing a static set of apps fixed by users.

Manuscript received November 8, 2012.

Manuscript revised June 6, 2013.

${ }^{\dagger}$ The authors are with the School of Computer Science, National University of Defense Technology, P.R. China.

${ }^{\dagger \dagger}$ The authors are with the School of Computer Science, McGill University, Canada.

*This research is supported by a research grant from the National Natural Science Foundation of China (No. 61170260). Thanks Xue Liu for his helpful advices.

a)E-mail: xiaxiao.cn@cs.mcgill.ca

DOI: 10.1587/transinf.E96.D.2860
2. We granularly mine the personalized usage patterns of mobile apps. In a novel approach, we then leverage such patterns to predict app usages and provide intelligent user interfaces using the live homescreen.

3. We implement the Personalized Live Homescreen system on the Android platform and empirically evaluate its effectiveness.

The PLH system is presented to address the app discovery problem in the face of intensive consumption of screen spaces on mobile devices. It also personalizes the usage of mobile devices and provides smart interactions with mobile apps. Consequently, the PLH system can improve the overall ubiquitous experiences for mobile users. Currently the system is implemented on the Android platform; however, the concept of live homescreen and the design and implementation methodologies of PLH can be easily applied to other mobile platforms with minimal modifications.

\section{Design and Implementation}

As shown in Fig. 1, the PLH system includes four components as follows:

Device monitor tracks the events of apps and the contexts of devices. It collects data for mining those essential patterns and drives the update of the live homescreen.

Pattern engine exploits and updates the app usage patterns in an online manner, i.e., it incrementally updates such patterns over time.

App predictor makes predictions based on the app usage patterns. It is triggered by the app usage or the change of the device context.

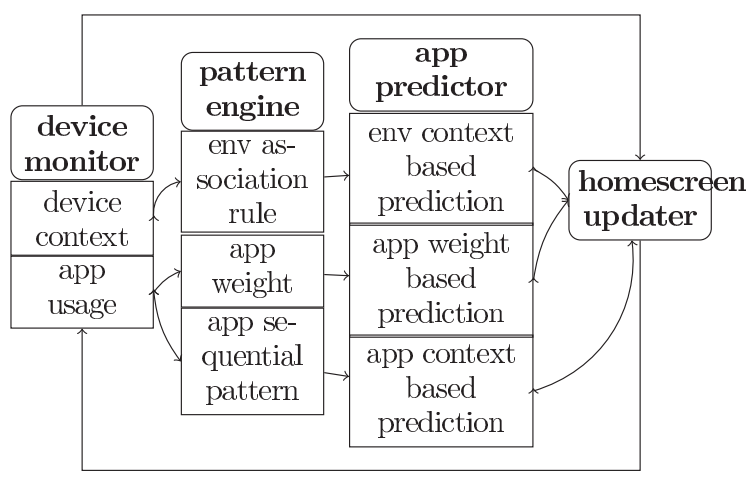

Fig. 1 Components of PLH system. 
Homescreen updater timely updates the shortcuts of predicted apps on the homescreen in both event-driven and context-driven manner.

\section{Patterns of App Usage}

The immense diversity among users in the usage of mobile apps [1]-[3] appeals to provide personalized experiences for users. To this end, we propose three patterns from representative perspectives: the app weight pattern, the app context pattern, and the env context pattern.

\subsection{App Weight Pattern}

The app weight patterns suggest the users' preferences on apps. They are introduced based on the diversity that has been observed among apps in their usage [4]. We define the app weight to measure the users' preferences. The app weight patterns are represented as a list of apps that are ranked by their weights in descending order. We propose three weighting schemes that result in different app weights:

- $w_{f}(i)$, the frequency weight, denotes the times that an app $i$ has been launched in a given period;

- $w_{o}(i)$, the overall weight, denotes the accumulative time that the app $i$ is active in a given period;

- $w_{a}(i)$, the average weight, denotes the average using time of the app $i$ after each launch in a given period.

\subsection{App Context Pattern}

The app context patterns reflect the dependencies between apps. They are proposed because the apps in mobile devices are not used independently [5] but sometimes in chains. We define the mining of app context patterns as the problem of mining the sequential patterns in the app usage history. It is equivalent to find all frequent sequences $\mathcal{S}$ in the sequence database $\mathcal{D}$ of all apps $\mathcal{A}$ with the minimum support threshold min_sup. The sequence database $\mathcal{D}=\left\{d_{1}, d_{2}, \cdots, d_{n}\right\}$ consists of sequential records of launched apps: $d_{i}=$ $<a p p_{i 1}, a p p_{i 2}, \cdots, a p p_{i k}>$, where $a p p_{i j}$ is launched earlier than app $_{i k}$ when $j<k$.

\subsection{Env Context Pattern}

The env context patterns reveal the dependencies of apps on their environments. Actually, strong dependencies have been observed between the app usages and their environment contexts [6]. We define the problem of mining the env context pattern as the problem of mining the association rules between the env contexts and apps. It is equivalent to find all association rules $\mathcal{R}$ in the event database $\mathcal{E}$ with the minimum support min_sup and the minimum confidence $\min _{-} c f d$. The database $\mathcal{E}=\left\{e_{1}, e_{2}, \cdots, e_{n}\right\}$ consists of app usage events that are combined with env context features and the using apps, e.g. $\{W i F i:$ on, $\cdots$, Location : home; App : webbrowser\}.

\section{Predictions of App Usage}

Based on the app usage patterns, we accordingly predict the apps to be used from three aspects. Such predictions are then integrated to serve as the final predictions that will be timely updated on the live homescreen.

\subsection{App Weight Based Prediction}

As shown in Fig. 2, we provide two kinds of app weight based predictions, i.e., the apps with largest weights $P_{\text {most }}$ and the apps with smallest weights $P_{\text {rare }}$. The $P_{\text {most }}$ directly reflects the users' preferences for apps, as we may assume that the mostly used apps are mostly to be used. The $P_{\text {rare }}$ are selected from the apps with smallest weights. They remind users of the apps that have been forgotten behind for a period of time. Such predictions relive previous preferences of users thus capture the interest drifting of them. It helps the discovery of all installed apps in personal devices.

\subsection{App Context Based Prediction}

As shown in Fig. 3, we maintain the app state pool to store a given number of apps that are recently used. Upon an app is launched, the system automatically updates the state pool and checks the accordance with app sequential patterns. Once the sequence of apps in the state pool meets one of the patterns, the corresponding app in that pattern is updated to the prediction set.

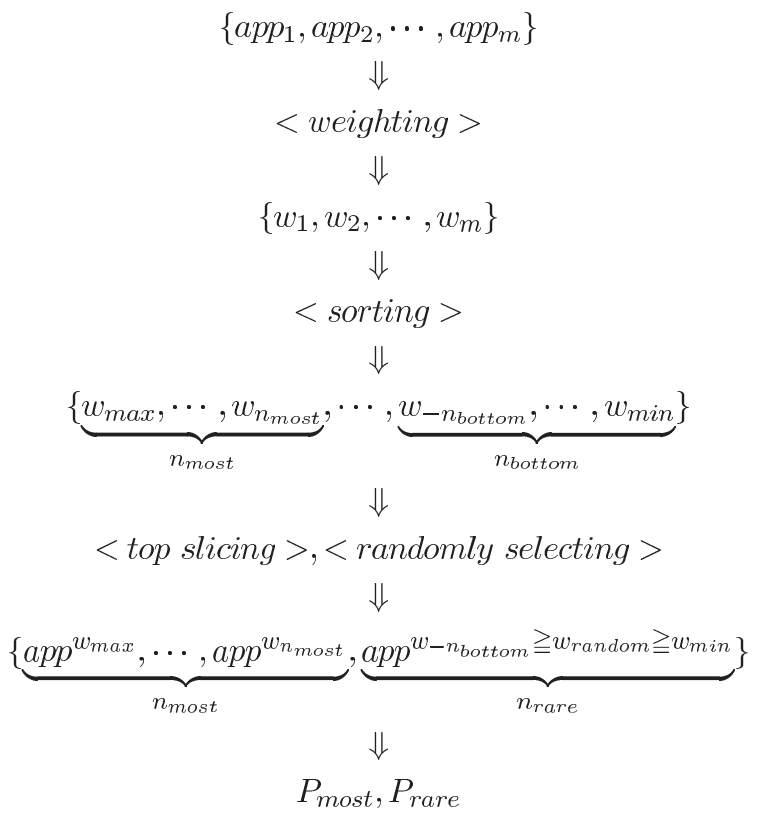

Fig. 2 App weight based prediction. After sorting apps by their usage weights, the system chooses the apps with top weights as $P_{\text {most }}$ predictions and selects some of the apps with smallest weights as $P_{\text {rare }}$ predictions. 


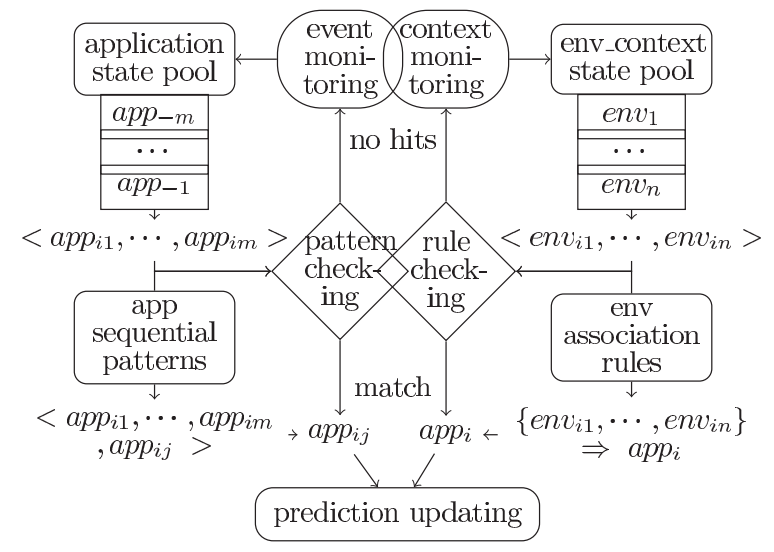

Fig. 3 App context based and env context based prediction. The device monitor keeps traces of app events and env contexts in corresponding state pools, based on which the system checks and matches the app sequential patterns or the env association rules to generate predictions.

\subsection{Env Context Based Prediction}

As shown in Fig. 3, we maintain the context state pool to store the states of the env contexts when apps are used, such as whether the WiFi is on. Upon time the env contexts are changed, the system automatically checks if the context state fits one of the association rules. Once one of such rules is met in its condition, the corresponding app in that rule is updated to the prediction set.

\subsection{Weighted Union of Predictions}

The above three predictions are united to derive the final predicting results. The union is dominated by two parameters the allocating number and the supplement priority. The allocating number is used since the space of the live homescreen of a mobile device is limited. We need to determine the number of apps extracted from each kind of predictions such that the total number extracted apps would not exceed the number of apps the live homescreen can support. The supplement priority is defined since the intersection of different kinds of predictions may be nonempty. Consequently, we need to supplement apps from some of the original predictions to fill the available space of the live homescreen.

\section{Evaluation}

In this section, we conduct evaluations to verify the effectiveness of our PLH system.

\subsection{Data Set}

Since the PLH system dedicates to the promotion of personalized experiences, we don't present evaluations in the scenarios of large-scale users. We have solicited 13 volunteers who are interested in our system and are willing to make contribution to the improvement of it. All of the participants have used the PLH system for more than 27 days
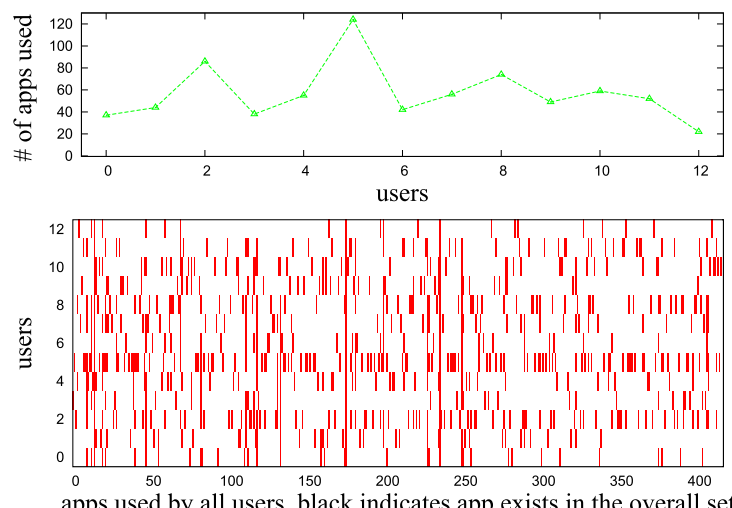

Fig. 4 Diversity of app usage among participants.

while permitting to provide their event logs. After all, we come out with 122547 log entries which include information of the app usages, the device contexts and the system predicting events.

Based on the log entries, Fig. 4 illustrates the number of apps for each user and their distributions in the overall app set. Such an experimental result verifies that notable diversity of app usages exists among the participants, although the number of them is not large and they are from the similar backgrounds. It thus further demonstrates the reliability of the data set for measuring the effectiveness of the PLH system.

\subsection{Evaluating Method}

Having the predicting and the usage history of apps, we define the hitrate of prediction to measure its accuracy, thus to measure the effectiveness of the PLH system. The hitrate is intuitively denoted as the ratio of the predictions which match the users' choices, that is,

$$
h=\left|N_{p} \cap N_{u}\right| /\left|N_{p}\right|,
$$

where the $N_{p}$ denotes the set of predicted apps and the $N_{u}$ denotes the set of used apps.

Moreover, since different devices provide homescreens of different spaces, we evaluate the PLH system by providing different number of apps shown on the live homescreen, i.e., the number of predicted apps.

\subsection{Evaluating Results}

Using the method above, we firstly evaluate the predictions of each separate app usage pattern and then the effectiveness of the whole system.

For the app weight patterns, Fig. 5 shows that predictions based on different weighting schemes achieve different hit rates. The predictions based on $w_{f}$ perform a little bit better than those based on $w_{o}$. Both of them overcome the predictions based on $w_{a}$ and achieve relative higher hit rates along with the increasing number of predicted apps. We can infer that the frequency and overall launch time of an app 


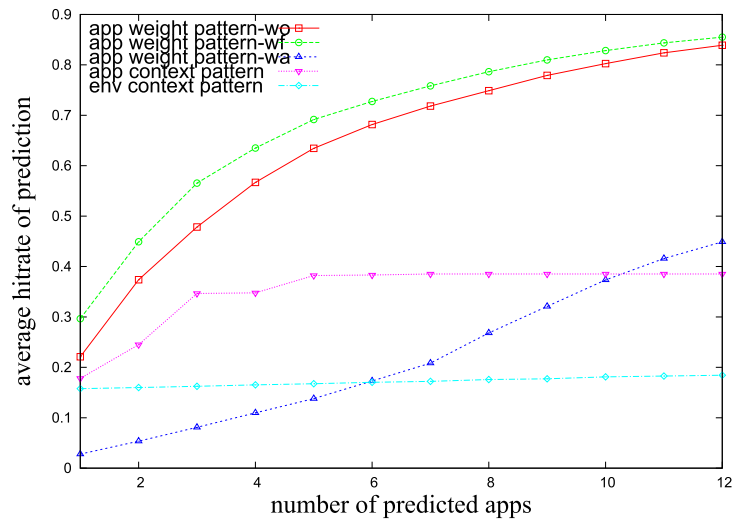

Fig.5 Average hit rate of predictions based on separate patterns. Evaluations are conducted by changing the number of apps shown on the PLH, i.e., the number of all the predicted apps.
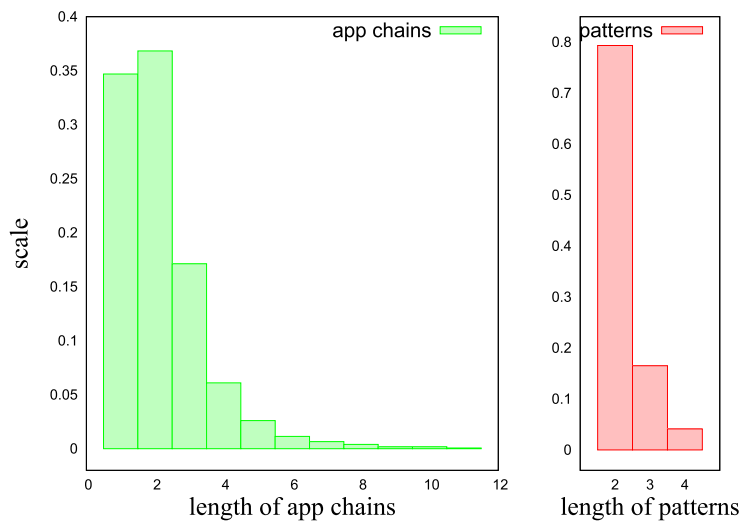

Fig. 6 Length distribution of the app context patterns and chains.

Table 1 Examples of the env context patterns from different users.

\begin{tabular}{c|c|c} 
Day, WiFi, Loc, Headset & App & Confidence \\
\hline$(3,0,2,0)$ & rovio.angrybirdsrio & 0.68 \\
$(3,1,1,0)$ & google.android.talk & 0.76 \\
$(1,1,1,0)$ & uc.browser & 0.87 \\
$(6,0,3,1)$ & android.phone & 0.89 \\
$(6,1,2,0)$ & tencent.qq & 0.93
\end{tabular}

indicate the users' preferences better.

For the app context patterns, Fig. 6 illustrates the length distribution of the app sequential chains and sequential patterns. We can see that although the patterns are short, there are long chains in app usages. It verifies the correlative dependencies among apps.

For the env context patterns, as listed in Table 1, there are meaningful dependencies of apps on the using context, where the context is defined as the combination of the day in the week (1:7), the WiFi state $(0: 1)$, the headset state $(0: 1)$, and the location ( 1 for work, 2 for home, 3 for outside).

Different patterns exhibit different effectiveness and variation over the number of predicted apps, as shown in Fig. 5. To be detailed, the app weight patterns mostly indicate the usages of apps. The app context patterns reach a stable performance with the growth of predicted apps. Pos-

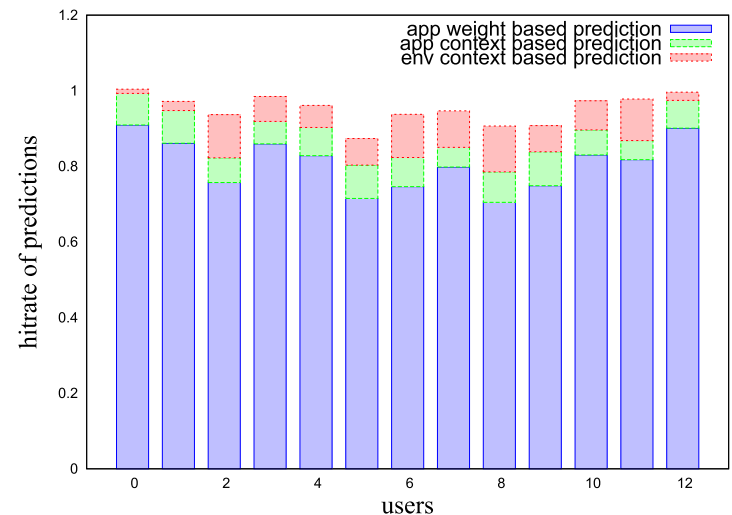

Fig. 7 Hit rate of the PLH system. Different patterns supplement each other and jointly contribute to the collaborative performance.

sible reasons may lay in that the apps which depend on the usages of other apps are within a certain range. The env context patterns do not perform better when providing more predictions. This may result from the reason that users tend not to launch many apps in particular situations.

Due to the diverse effectiveness of different predictions, we combine the power of them to integrate the whole system. As shown in Fig. 7, the PLH system provides distinguish effectiveness for the app prediction. The root cause is that the three predictions supplement each other and thus jointly contribute to the collaborative performance.

\section{Related Work}

On the analysis of app usage for mobile users, the research community has conducted valuable investigations [1], [4][6]. However, they mainly focus on the analysis of large scale of devices, users or apps, in contrast of the fine-grained mining of personal usage patterns, addressed in this paper.

Applications leveraging the usage of mobile apps have already been presented [7]-[9]. However, they mainly concentrate on those apps that have not be installed by users, instead of promoting the user experiences with apps that have been installed. Additionally, they did not give attention to providing intelligent user interfaces.

\section{Conclusion}

We design, implement and evaluate the PLH system, which identifies the potential of providing personalized intelligent interface by leveraging the app usage patterns.

\section{References}

[1] H. Falaki, R. Mahajan, S. Kandula, D. Lymberopoulos, R. Govindan, and D. Estrin, "Diversity in smartphone usage," MobiSys '10, pp.179194, 2010.

[2] E.A. Oliver and S. Keshav, "An empirical approach to smartphone energy level prediction," UbiComp'11, pp.345-354, 2011.

[3] A.K. Dey, K. Wac, D. Ferreira, K. Tassini, J.H. Hong, and J. Ramos, "Getting closer: An empirical investigation of the proximity of user to their smart phones," UbiComp'11, pp.163-172, 2011. 
[4] Q. Xu, J. Erman, A. Gerber, Z. Mao, J. Pang, and S. Venkataraman, "Identifying diverse usage behaviors of smartphone apps," IMC'11, pp.329-344, 2011.

[5] M. Böhmer, B. Hecht, J. Schöning, A. Krüger, and G. Bauer, "Falling asleep with angry birds, facebook and kindle: A large scale study on mobile application usage,’ MobileHCI '11, pp.47-56, 2011.

[6] T.M.T. Do, J. Blom, and D. Gatica-Perez, "Smartphone usage in the wild: A large-scale analysis of applications and context,' ICMI'11, pp.353-360, 2011.
[7] Y. Chon, E. Talipov, H. Shin, and H. Cha, "Mobility prediction-based smartphone energy optimization for everyday location monitoring," SenSys '11, pp.82-95, 2011.

[8] H. Shin, Y. Chon, K. Park, and H. Cha, "FindingMiMo: Tracing a missing mobile phone using daily observations," MobiSys '11, pp.2942, 2011.

[9] B. Yan and G. Chen, "Appjoy: Personalized mobile application discovery,” MobiSys '11, pp.113-126, 2011. 